\title{
Cooperative Spectrum Sensing for Heterogeneous Sensor Networks Using Multiple Decision Statistics
}

\author{
Shree Krishna Sharma, Symeon Chatzinotas, and Björn Ottersten \\ SnT - securityandtrust.lu, University of Luxembourg, L-2721, Luxembourg \\ Email:\{shree.sharma, symeon. chatzinotas, bjorn.ottersten\}@uni.lu
}

\begin{abstract}
The detection of active Primary Users (PUs) in practical wireless channels with a single Cognitive Radio (CR) sensor is challenging due to several issues such as the hidden node problem, path loss, shadowing, multipath fading, and receiver noise/interference uncertainty. In this context, Cooperative Spectrum Sensing (CSS) is considered a promising technique in order to enhance the overall sensing efficiency. Existing CSS methods mostly focus on homogeneous cooperating nodes considering identical node capabilities, equal number of antennas, equal sampling rate and identical Signal to Noise Ratio (SNR). However, in practice, nodes with different capabilities can be deployed at different stages and are very much likely to be heterogeneous in terms of the aforementioned features. In this context, we propose a novel decision statistics-based centralized CSS technique using the joint Probability Distribution Function (PDF) of the multiple decision statistics resulting from different processing capabilities at the sensor nodes and compare its performance with various existing cooperative schemes. Further, we provide a design guideline for the network operators to facilitate decision making while upgrading a sensor network.
\end{abstract}

Key words: Cooperative Spectrum Sensing, Cognitive Radio, Joint PDF, Heterogeneous Sensor Networks

\section{Introduction}

Cognitive Radio (CR) communications is considered a promising solution in order to address the spectrum scarcity problem caused by the high demand of data rates and current frequency allocation policies. The most commonly used spectrum sharing paradigms in the literature are interweave, underlay, and overlay $[1,2]$. Out of several spectrum awareness techniques for enabling these paradigms, Spectrum Sensing (SS) is an important mechanism in order to exploit the spectral gaps in the underutilized primary spectrum so that they can be used by Secondary Users (SUs) in order to enhance the overall spectral efficiency of the system. Several SS techniques such as Matched filter, Energy Detection (ED), Cyclostationary Detection (CD), Autocorrelation-based detection (AD), Eigenvalue-based Detection, etc. have been proposed in the literature for CR systems [3]. These techniques have different operational requirements, advantages 
and disadvantages from the practical perspectives and can be broadly categorized into: (i) knowledge-aware, (ii) semi-blind, (ii) blind SS techniques $[4,5]$.

In practical wireless fading channels, the SS efficiency of the aforementioned techniques may be degraded due to the hidden node problem, path loss, shadowing, multipath fading and receiver noise/interference uncertainty issues. In this context, Cooperative Spectrum Sensing (CSS) has been considered as a promising approach $[6,7,8]$. The main concept behind CSS is to enhance the sensing performance by exploiting the observations captured by spatially located CR users. The CSS gain is achieved by sharing the information gathered by the cooperating users, thus making the combined decision more reliable than the individual decisions. Despite the its several advantages [6], CSS requires a control channel for each cooperating node to report its sensed information to the Fusion Center (FC) and this channel is usually bandwidth limited. Thus, cooperation burden can be a critical issue from a practical perspective. In this context, we are interested in studying the decision statistics-based centralized CSS which can reduce the signalling burden compared to the one of sample-based CSS and at the same time achieves the desired level of sensing performance.

Most of the existing CSS literature considers a CR network with homogeneous sensor nodes and assumes identical capabilities, equal number of antennas, equal sampling rate and identical received Signal to Noise Ratio (SNR) for all the cooperating nodes. However, in practice, the nodes with different capabilities can be deployed at different stages and are very much likely to be heterogeneous in terms of the aforementioned features. In this context, it's an important challenge to investigate suitable CSS techniques which can provide better sensing performance and low signalling overhead in heterogeneous environments. Further, most of the existing decision and data fusion techniques in the CSS context use a single type of detector (ED in many cases) as local and CSS mechanisms. However, in heterogeneous environments, different nodes can employ separate decision statistics since they may have different capabilities. The issue of data fusion considering different decision statistics for CSS has not been addressed in the literature.

To address the aforementioned issues, this paper investigates the combination of ED and eigenvalue-based decision statistics in order to achieve reliable sensing in heterogeneous environments. More specifically, we propose a novel CSS technique based on the joint Probability Distribution Function (PDF) of multiple decision statistics. We consider that these multiple decision statistics arise from the different processing capabilities of the heterogeneous cooperating nodes. We evaluate and compare the detection performance of the proposed approach with the existing cooperative approaches. Moreover, we provide a design guideline for the network operators to facilitate decision making while upgrading a sensor network.

The remainder of this paper is structured as follows. Section 2 presents the system and signal models, and further describes the considered local and cooperative detection techniques. Section 3 proposes a novel CSS approach for the considered heterogeneous environment. Section 4 evaluates and compares the 
performance of the proposed approach with several existing approaches with the help of numerical results. Finally, Section 5 concludes the paper.

\section{System and Signal Model}

We consider a large scale CR network consisting of $N_{c}$ number of heterogeneous cooperating nodes which communicate with a FC as depicted in Fig. 1. The considered heterogeneous environment is motivated from the real world practical scenarios. If we consider the deployment of nodes in a sensor network, different nodes may be deployed at different stages as the available technology evolves. It is in general both impractical and wasteful to replace all the existing nodes with the new nodes while implementing a new technology. The heterogeneity of the nodes can be in the form of capability of nodes, number of samples acquired during the sensing time (sampling rate and sampling time can be different for different nodes), number of antennas equipped in the nodes and the received PU SNR. For simplicity of analysis in this paper, we assume the same received PU SNR at each cooperating node, and fixed number of samples. Further, we consider the following two categories of nodes on the basis of their capabilities: (i) existing nodes which are capable of performing a simple sensing algorithm such as ED (We call these nodes the first generation nodes.), and (ii) new sensor nodes which are capable of performing advanced sensing techniques such as eigenvalue-based algorithms (We call these nodes the second generation nodes).

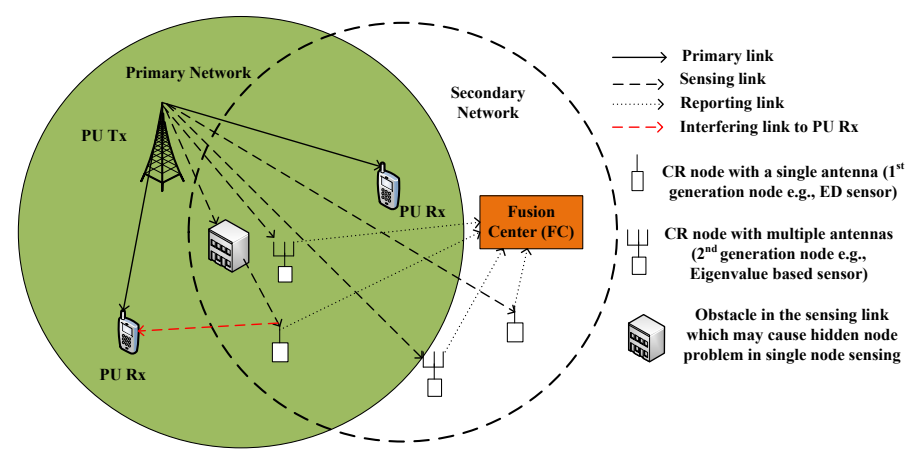

Fig. 1. Schematic of the considered CSS scenario with heterogeneous nodes

Let $N=\left\lceil\tau f_{s}\right\rceil$ be the number of observations collected by each node in the time duration of $\tau, f_{s}$ being the sampling frequency. We denote the hypothesis of the PU absence and the PU presence by $H_{0}$ and $H_{1}$ respectively. When each cooperating node performs local sensing independently, the SS problem can be written as

$$
\begin{aligned}
& y_{k}(n)=z_{k}(n), \quad H_{0} \\
& y_{k}(n)=h_{k} s(n)+z_{k}(n), \quad H_{1}
\end{aligned}
$$


where $s(n)$ is the $n$th sample of the transmitted PU signal, $h_{k}$ denotes the complex gain of the channel between the PU and the $k$ th node, and $z_{k}(n)$ is the Additive White Gaussian Noise (AWGN) at the receiver of the $k$ th cooperating node.

We assume that the sensing channel remains constant during the period of sensing and the transmitted PU symbols are independent and identically distributed (i.i.d.) complex circularly symmetric (c.c.s.) symbols. Further, we assume that the reporting channels are ideal as considered in various literature $[4,9]$. The decision statistic used for testing the above hypothesis problem depends on the employed detection technique. For a detection technique based on a single decision statistic, let us denote by $T$, the probability of detection $\left(P_{d}\right)$ and the probability of false alarm $\left(P_{f}\right)$ can be calculated as: $P_{d}=\operatorname{Pr}\left(T>\lambda \mid H_{1}\right)$, and $P_{f}=\operatorname{Pr}\left(T>\lambda \mid H_{0}\right)$, where $\operatorname{Pr}(\cdot)$ denotes the probability.

1. Sensing Techniques: In the CSS, the local nodes may employ any one of the sensing techniques such as $\mathrm{ED}, \mathrm{CD}, \mathrm{AD}$, eigenvalue detector, etc. in order to capture the information about the presence or absence of the PU signal. In this paper, we consider the following two detection techniques in the considered heterogeneous environment.

i. Energy Detection: For the ED detector, the decision statistic for the $k$ th cooperating node is given by $T_{k}=\frac{1}{N} \sum_{n=1}^{N} y_{k}(n)^{2}$. For the Circularly Symmetric Complex Gaussian (CSCG) noise with variance $\sigma_{z}^{2}$, the expression for $P_{f}$ can be written as [10]: $P_{f}(\lambda, \tau)=Q\left(\left(\frac{\lambda}{\sigma_{z}^{2}}-1\right) \sqrt{\tau f_{s}}\right)$, where $Q($.$) is the$ complementary distribution function of the standard Gaussian random variable. Similarly, under the $H_{1}$ hypothesis, the expression for $P_{d}$ is given by; $P_{d}(\lambda, \tau)=Q\left(\left(\lambda / \sigma_{z}^{2}-\gamma_{\mathrm{p}}-1\right) \sqrt{\frac{\tau f_{s}}{2 \gamma_{p}+1}}\right)$, where $\gamma_{p}$ is the received SNR of the primary signal measured at the CR receiver. To enhance the sensing efficiency in fading channels, different receive diversity schemes have been considered in the literature $[11,12,13]$.

ii. Eigenvalue-based Detection: In this approach, different eigenvalue properties of the received signal's covariance matrix can be exploited to perform sensing. Several eigenvalue-based sensing and SNR estimation techniques have been proposed in the literature exploiting the properties of Wishart random matrices $[4,14,15,16]$. The main advantage of the eigenvalue-based approach in practical scenarios is that it does not require any prior information about the PU's signal and the channel.

After collecting $N$ samples using $M$ receive dimensions, we form the $M \times N$ received signal matrix $\mathbf{Y}$ and define sample covariance matrices of the received signal and the noise as: $\mathbf{R}_{\mathbf{Y}}(N)=\frac{1}{N} \mathbf{Y} \mathbf{Y}^{H}$ and $\mathbf{R}_{\mathbf{Z}}(N)=\frac{1}{N} \mathbf{Z} \mathbf{Z}^{H}$. Under the $H_{0}$ hypothesis, $\mathbf{R}_{\mathbf{Y}}(N)=\mathbf{R}_{\mathbf{Z}}(N)$. By using different eigenvalue properties of $\mathbf{R}_{\mathbf{Y}}(N)$ such as Maximum Eigenvalue (ME) [17], Signal Condition Number (SCN) [4, 15], Scaled Largest Eigenvalue (SLE) [14], etc., the presence or absence of the PU signal can be decided.

2. Data/Decision Fusion Schemes: Based on the employed cooperative decision mechanism, the nodes can forward one of the following parameters (i) hard decision (single bit), (ii) decision statistics, (iii) quantized decision fusion (multiple 
bits), and (iv) all the samples (measurements) collected over the sensing duration. Based on these parameters, the existing fusion mechanisms in the FC can be categorized into the following.

a. Hard Decision Fusion: In this scheme, each node forwards a single bit decision to the FC i.e., 1 for the PU signal presence case and 0 for the PU signal absence case. The requirement of the limited bandwidth is the main advantage of this approach. The FC can employ any one of the "AND", "OR", or majority decision rules. The expressions for $P_{d}$ and $P_{f}$ with the "OR" decision rule are given by [9]; $P_{d}=1-\prod_{m=1}^{N_{c}}\left(1-P_{d, m}\right)$, and $P_{f}=1-\prod_{m=1}^{N_{c}}\left(1-P_{f, m}\right)$, where $P_{d, m}$ and $P_{f, m}$ being the $P_{d}$ and the $P_{f}$ of the $m$ th sensing node while using the local sensing technique. Further, the expressions for $P_{d}$ and $P_{f}$ using the "AND" decision rule can be written as: $P_{d}=\prod_{m=1}^{N_{c}} P_{d, m}$, and $P_{f}=\prod_{m=1}^{N_{c}} P_{f, m}$.

b. Decision Statistics-based Soft Data Fusion: In this approach, the nodes forward the decision statistics i.e., energy in the ED context, without performing any decision and the FC makes the decision by combining them using different combining methods such as Equal Gain Combining (EGC), Maximum Ratio Combining (MRC), and Selection Combining (SC) [18]. This method provides better performance than the hard combination schemes but requires a larger bandwidth for the reporting channels [8].

c. Quantized (soft hardened) Decision Fusion: In this scheme, the nodes send the local decision in the form of multiple bits instead of a single bit in the hard decision fusion scheme and the FC provides different weights to these decisions while making the final decision. This method provides better detection performance than the hard decision scheme at the expense of the signalling overhead [9].

d. Samples-based Soft Data Fusion: In this approach, the nodes forward all the samples captured during the period of sensing. The main disadvantage of this technique is that it requires high bandwidth of the reporting links. Although decision statistics-based data fusion and sample-based data fusion seem to provide similar performance in the ED context (as illustrated in Section 4), they may provide different performance for other decision statistics.

While comparing the existing SS techniques, it can be noted that the ED technique is simple to implement but is susceptible to noise variance uncertainty [19]. This drawback can be addressed by using blind eigenvalue-based techniques such as SCN, SLE, John's Detection (JD) method, Spherical Test (ST) detector, etc [5]. However, these techniques are complex in comparison to the ED technique and require an Eigenvalue Decomposition (EVD) operation in order to calculate the decision statistics. Assuming that newly deployed nodes are capable of performing EVD operation and the existing nodes can only perform the ED detection, the research problem is how to make reliable sensing decision by exploiting different decision statistics originating in heterogeneous nodes of a CR network. In this context, we apply the marginal approximation-based approach to calculate decision thresholds based on the joint PDF of multiple decision statistics. This process is carried out at the beginning of the system operation and fixed thresholds (e.g., based on the look-up tables) can be used while com- 
bining the instantaneous decision statistics forwarded by the cooperating nodes.

\section{Proposed Cooperative Sensing Method}

1. Multivariate Preliminaries: The probability density function of a single normal random variable $x$ is given by

$$
f(x)=\frac{1}{\sqrt{2 \pi} \sigma} \exp \left(-\frac{1}{2 \sigma^{2}}(x-\mu)^{2}\right),
$$

where $\mu$ and $\sigma^{2}$ are mean and the variance of the distribution respectively. The density functions of two random variables $x_{1}$ and $x_{2}$ which are correlated by a correlation coefficient $\rho$ is given by

$f\left(x_{1}, x_{2}\right)=\frac{1}{2 \pi \sigma_{1} \sigma_{2} \sqrt{1-\rho^{2}}} \exp \left(-\frac{\left(x_{1}-\mu_{1}\right)^{2}}{2 \sigma_{1}^{2}\left(1-\rho^{2}\right)}+\frac{\left(x_{2}-\mu_{2}\right)^{2}}{\sigma_{2}^{2}}-\frac{2 \rho\left(x_{1}-\mu_{2}\right)\left(x_{2}-\mu_{2}\right)}{\sigma_{1} \sigma_{2}}\right)$.

The set of points for which the values of $x_{1}$ and $x_{2}$ give the same value for the density function $f\left(x_{1}, x_{2}\right)$ can be defined as an isodensity contour and is given by $[20]$

$$
\frac{\left(x_{1}-\mu_{1}\right)^{2}}{\sigma_{1}^{2}}+\frac{\left(x_{2}-\mu_{2}\right)^{2}}{\sigma_{2}^{2}}-2 \rho \frac{\left(x_{1}-\mu_{1}\right)\left(x_{2}-\mu_{2}\right)}{\sigma_{1} \sigma_{2}}=P .
$$

The above equation defines an ellipse with the centroid $\left(\mu_{1}, \mu_{2}\right)$, which is the locus of points representing the combinations of the values of $x_{1}$ and $x_{2}$ with the same probability, defined by the constant $P$. For various values of $P$, we can obtain a family of concentric ellipses having different cross sections of the density surface with planes at various elevations. The angle joining the axis center with the centroid of the distribution, let us denote by $\theta$, is independent of the value of $P$ and depends on the values of $\sigma_{1}, \sigma_{2}$ and $\rho$. The steepness of this line depends on the correlation i.e., the higher the correlation, the steeper is the line.

$$
f(\mathbf{x})=(2 \pi)^{-1} \Sigma^{-\frac{1}{2}} e^{-\frac{1}{2} \mathbf{X}^{\prime} \Sigma^{-1} \mathbf{x}},
$$

where $\mathbf{X}=\mathbf{x}-\mu$ and $\Sigma=\left(\begin{array}{cc}\sigma_{1}^{2} & \rho \sigma_{1} \sigma_{2} \\ \rho \sigma_{1} \sigma_{2} & \sigma_{2}^{2}\end{array}\right)$. The bivariate distribution function (5) can be generalized as $n$-variate distribution in the following way [20].

$$
f(\mathbf{x})=(2 \pi)^{-n / 2} \Sigma^{-\frac{1}{2}} e^{-\frac{1}{2} \mathbf{X}^{\prime} \Sigma^{-1} \mathbf{X}} .
$$

In (6), an ellipsoid is formed for a fixed value of density $f(\mathbf{x})$. It should be noted that $\mathbf{X}^{\prime} \Sigma^{-1} \mathbf{X}$ is a chi-square $\left(\chi^{2}\right)$ variate and the inequality $\mathbf{X}^{\prime} \Sigma^{-1} \mathbf{X} \leq \chi^{2}$ defines any point within the ellipsoid.

2. Signal Detection using Multiple Decision Statistics: Despite the important application of joint PDF for PU signal detection, it has received limited attention in the CR literature. The contribution in [21] has proposed a method of constructing a joint PDF under the $H_{1}$ hypothesis assuming that the joint PDF under the $H_{0}$ hypothesis is known. Recently, authors in [22] have exploited the moments of 
joint and marginal distributions of extreme eigenvalues in order to find out the decision threshold of a SCN-based detector. In [22], the joint PDF of the maximum and the minimum eigenvalues is approximated by a dependent Gaussian distribution function. The signal detection using multiple decision statistics can be considered as a generalization of the univariate representation [23]. Instead of being represented as a point on a line in the univariate case, a multivariate observation becomes a point in multi-dimensional space. The signal detection using multiple decision statistics can be performed either using the multivariate PDF or multivariate Cumulative Distribution Function (CDF) evaluated under the $H_{0}$ hypothesis. For example, for the signal detection based on bivariate CDF, the instantaneous test pair $\left(T_{1}, T_{2}\right)$ can be checked whether it lies within the contour plot of the bivariate PDF corresponding to a predetermined $P_{f}$ or not in order to decide on the presence or absence of the PU signal. Similarly, for the signal detection based on the bivariate PDF, the decision can be taken by testing the probability of instantaneous pair $\left(T_{1}, T_{2}\right)$ lying within an ellipsoid corresponding to a target $P_{f}$ as mentioned before. In Section 4, we present results evaluated based on the bivariate PDF-based approach using energy and eigenvalue-based decision statistics.

Let $f\left(T_{1}, T_{2}\right)$ and $F\left(T_{1}, T_{2}\right)$ denote the joint PDF and joint $\mathrm{CDF}$ of two decision statistics $T_{1}$ and $T_{2}$. Then the expression for $P_{f}$ can be written as

$$
P_{f}=1-F\left(\lambda_{1}, \lambda_{2}\right)=1-\int_{-\infty}^{\lambda_{1}} \int_{-\infty}^{\lambda_{2}} f\left(T_{1}, T_{2}\right) d T_{2} d T_{1},
$$

where $\lambda_{1}$ and $\lambda_{2}$ correspond to thresholds obtained from the joint distribution of $T_{1}$ and $T_{2}$, respectively. From (7), it can be observed that we need to find two decision thresholds unlike a single decision threshold in the univariate signal detection problem. Therefore, the main issue in the bivariate detection problem is the calculation of decision threshold pair $\left(\lambda_{1}, \lambda_{2}\right)$ in order to satisfy the desired probability of false alarm constraint. While applying the univariate decision rule, we should be able to calculate the threshold pair using the following expression

$$
\left(\lambda_{1}, \lambda_{2}\right)=F^{-1}\left(1-P_{f}\right),
$$

where $F^{-1}$ denote the inverse of the joint $\mathrm{CDF}$ of the decision statistics $T_{1}$ and $T_{2}$. If we can find the unique values of $\lambda_{1}$ and $\lambda_{2}$ corresponding to the inverse of the joint CDF, we can find the optimum pair of threshold to take the decision. However, there exist multiple pairs of $\left(\lambda_{1}, \lambda_{2}\right)$ which yield the same $P_{f}$. Therefore, the bivariate detection problem becomes complex in comparison to the univariate detection problem. To address this issue, different approximation methods can be exploited in order to obtain the approximated threshold pair. In this paper, we focus on marginal approximation method ${ }^{1}$ in order to derive the decision thresholds for two decision statistics. It should be noted that in our case, the decision statistics are independent random variables and the joint PDF

\footnotetext{
${ }^{1}$ Investigating other suitable approximation methods based on the joint PDF of multiple decision statistics is our ongoing work.
} 
of two independent random variables is equal to the product of their marginal distributions.

Using marginal approximation, the values of decision thresholds $\lambda_{1 m}$ and $\lambda_{2 m}$ can be obtained using $\lambda_{1 m}=F_{T_{1}}^{-1}\left(1-P_{f}\right)$ and $\lambda_{2 m}=F_{T_{2}}^{-1}\left(1-P_{f}\right)$, where $F_{T_{1}}$ and $F_{T_{2}}$ are marginal CDFs of $T_{1}$ and $T_{2}$, respectively and can be obtained from their joint $\operatorname{PDF} f\left(T_{1}, T_{2}\right)^{2}$ in the following way

$$
\begin{aligned}
& F_{T_{1}}\left(\lambda_{1}\right)=\lim _{\lambda_{2} \rightarrow \infty} \int_{-\infty}^{\lambda_{1}} \int_{-\infty}^{\lambda_{2}} f\left(T_{1}, T_{2}\right) d T_{2} d T_{1} . \\
& F_{T_{2}}\left(\lambda_{2}\right)=\lim _{\lambda_{1} \rightarrow \infty} \int_{-\infty}^{\lambda_{1}} \int_{-\infty}^{\lambda_{2}} f\left(T_{1}, T_{2}\right) d T_{2} d T_{1} .
\end{aligned}
$$

After obtaining marginal thresholds $\lambda_{1 m}$ and $\lambda_{2 m}$, we apply the following two different decision rules in order to take the decision about the presence or the absence of the PU signal.

i. Logical OR Rule: In this method, the expressions for $P_{d}$ and $P_{f}$ can be written as $\mathrm{P}_{d}=\operatorname{Pr}\left(T_{1}>\lambda_{1 m} \mid H_{1}\right.$ OR $\left.T_{2}>\lambda_{2 m} \mid H_{1}\right)$,

$P_{f}=\operatorname{Pr}\left(T_{1}>\lambda_{1 m} \mid H_{0}\right.$ OR $\left.T_{2}>\lambda_{2 m} \mid H_{0}\right)$. ii. Logical AND rule: In this scheme, the expressions for $P_{d}$ and $P_{f}$ are given by $\mathrm{P}_{d}=\operatorname{Pr}\left(T_{1}>\lambda_{1 m} \mid H_{1}\right.$ AND $\left.T_{2}>\lambda_{2 m} \mid H_{1}\right)$, $P_{f}=\operatorname{Pr}\left(T_{1}>\lambda_{1 m} \mid H_{0}\right.$ AND $\left.T_{2}>\lambda_{2 m} \mid H_{0}\right)$.

\section{Numerical Results}

[a]

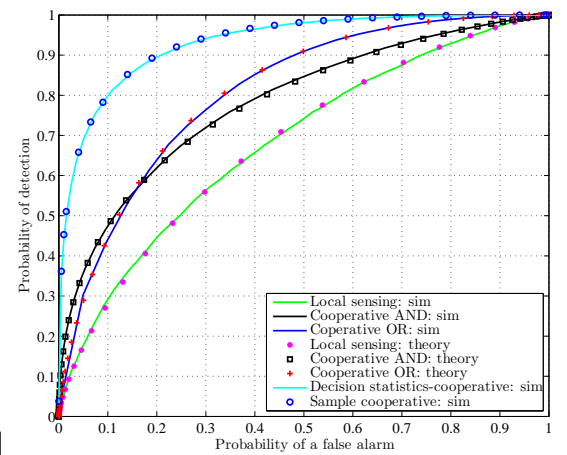

Fig. 2. (a) Performance comparison of different cooperative schemes in an AWGN channel $(K=1$, $N=50, N_{c}=10, S N R=-10 \mathrm{~dB}$ ), (b) Performance comparison of the proposed approach with other cooperative schemes considering different processing capabilities and equal number of antennas in a Rayleigh fading channel $\left(K=4, N=50, N_{c}=10, S N R=-12 \mathrm{~dB}\right)$.

\subsection{Comparison of Existing CSS Schemes}

In this subsection, we compare the sensing performance of different hard decision and soft data fusion techniques in terms of their Receiver Operating Characteristics (ROC) i.e., $P_{d}$ versus $P_{f}$ plot. Figure 2(a) presents the ROC comparison of

${ }^{2}$ In the considered heterogeneous environment, we assume that only joint PDF of decision statistics is available at the FC. 
different techniques with parameters (Number of antennas in each node $(K)=1$, $N=50, N_{c}=10, S N R=-10 \mathrm{~dB}$ ). Further, we present theoretical results for hard fusion and local sensing in order to validate our approach. In this experiment, we use an AWGN channel and the ED as the local sensing technique. Further, we use the EGC-based soft data fusion technique, and "OR" and "AND" rules-based decision fusion techniques for the comparison purpose. It should be noted that in all the simulated results, the distribution of decision statistics under the $H_{0}$ hypothesis was computed by accumulating decision statistics over $10^{4}$ noise only realizations and then the decision threshold was calculated using the constructed distribution for a certain $P_{f}$ value. From the figure (Fig. 2(a)), it can be noted that the CSS with "AND" and "OR" rules-based decision fusion schemes perform better than the local ED sensing and the performance of "AND"-based decision fusion technique is better at the lower $P_{f}$ values than the "OR"-based combining method as depicted in the literature [9]. Additionally, we can note that the CSS with EGC i.e., decision statistics-based soft data fusion performs better the hard decision fusion techniques. Another important observation from Fig. 2(a) is that the ED decision statistics-cooperative provides similar performance as that of the ED sample-cooperative scheme.

\subsection{Performance Evaluation of the Proposed Approach}

In this subsection, we compare the performance of the proposed approach with the existing CSS schemes considering heterogeneity firstly in terms of processing capabilities, and then in terms of processing capabilities and the number of antennas as described below.

1. Heterogeneity in terms of Processing Capabilities: In order to compare the performance of the proposed approach with different schemes, we consider the following cases: (i) ED local sensing, (ii) Proposed joint PDF-based approach with ORing of the ME and energy statistics, obtained from the marginal approximation, in which half of the nodes forward energy and other half forward ME decision statistics, (iii) Proposed joint PDF-based approach with ANDing of the ME and energy, obtained from the marginal approximation, and the decision statistics forwarding as in case (ii), (iv) Proposed joint PDF-based approach with ORing of the SLE and energy, obtained from the marginal approximation, in which half of the nodes forward energy and other half forward SLE decision statistics, (v) ED sample-cooperative scheme in which all nodes forward the samples, and then the FC calculates the energy decision statistics and takes decision based on the threshold calculated under the $H_{0}$ hypothesis, (vi) Cooperative OR-based hard decision fusion with each node employing an ED sensor, (vii) Cooperative AND-based hard decision fusion with each node employing an ED sensor, and (viii) ME sample-cooperative in which all nodes forward the samples, and the FC makes decision based on ME decision statistics.

Figure 2(b) presents ROC curves in a Rayleigh fading channel for the aforementioned schemes with parameters $\left(K=4, N=50, N_{c}=10, S N R=-12\right.$ $\mathrm{dB})$. From the results, it can be depicted that the proposed approach provides better performance than the local sensing and the considered hard decision fusion 
schemes. Further, it can be noted that the proposed approach with the ORing of the ME and energy decision statistics provides slightly better performance than the ANDing approach, and the performance of the proposed approach with ORing of SLE and energy decision statistics is worse than that of the ORing and Anding schemes with energy and the ME decision statistics. During simulation, it has been noted that the ED sample-cooperative and the ED decision statistics-cooperative provide similar sensing performance as noted in Fig. 2(a). In addition, From Fig. 2(b), it is noted that the performance of the proposed approach with the ORing of the ME and energy decision statistics is slightly worse than the ED sample-cooperative scheme. However, the proposed scheme has low signalling burden compared to the sample-cooperative ED scheme as well as the decision statistics-based ED scheme since the transmission of the eigenvalue-based decision statistics e.g., the ME, requires less bandwidth than that of forwarding all the samples and the total energy.

2. Heterogeneity in terms of Processing Capabilities and Number of Antennas:

[a]

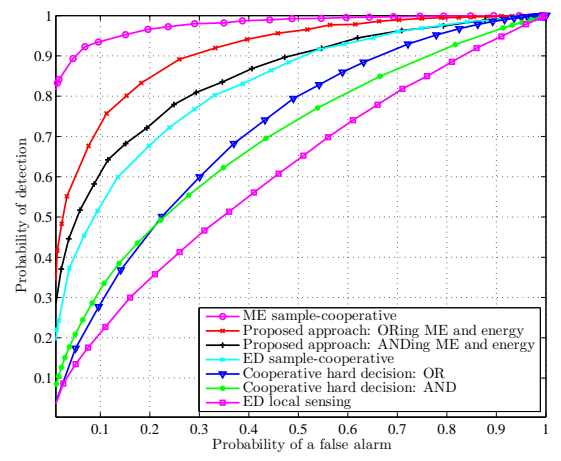

[b]

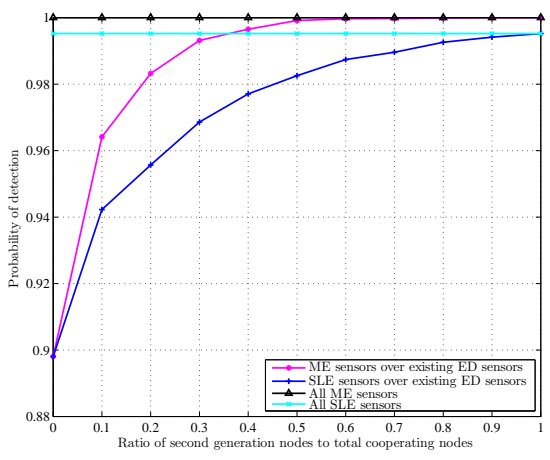

Fig. 3. (a) Performance comparison of different cooperative schemes in an AWGN channel $(K=1$, $N=50, N_{c}=10, S N R=-10 \mathrm{~dB}$ ), (b) $P_{d}$ versus the ratio of the second generation nodes to the total number of nodes $\left(K=1\right.$ for ED sensors and $K=4$ for ME/SLE sensors, $N=100, N_{c}=10$, $\left.S N R=-10 \mathrm{~dB}, P_{f}=0.1\right)$.

The results in Fig. 2(b) consider the heterogeneity of nodes in terms of their processing capabilities but assume the same number of antennas for all the sensors. To better illustrate the performance in the considered heterogeneous scenario, we present ROC curves for the aforementioned schemes in Fig. 3(a) with parameters $\left(K=1\right.$ for ED sensor and $K=4$ for ME sensor, $N=50, N_{c}=10$, $S N R=-12 \mathrm{~dB}$ ). It can be noted that the proposed approach with the ORing and the ANDing of the ME and the energy decision statistics perform better than the sample-cooperative ED scheme in the considered scenario. Further, from Fig. 3(a), it can be noted that the ME sample-cooperative provides the best performance but it requires a large signalling overhead for forwarding all the samples to the FC.

3. Discussion on Cooperative Signalling Burden: As mentioned earlier, in a sample-based CSS scheme, cooperating nodes have to forward all the samples collected during the period of sensing to the FC. Subsequently, the FC may em- 
ploy any of the SS schemes to take the decision about the presence or absence of the active PU. However, in the considered decision statistics-based approach, the cooperating nodes need to forward only the decision statistics i.e., energy value for the ED. It can be noted that the number of quantized bits required to deliver all the samples to the FC is significantly higher than the number of bits required to send only the decision statistics. In the proposed approach using multiple decision statistics, some of the nodes employ the eigenvalue based decision statistics and the rest employ the energy. Although hard decision fusion scheme has low signalling burden compared to the proposed one, its sensing performance is far worse than that of the proposed one as noted in Figs. 2(b) and 3(a). From the results presented in Fig. 3(a), it can be concluded that in one hand, the proposed approach saves a lot of signalling resources since it requires the transmission of only decision statistics instead of all samples, and on the other hand, it provides better sensing performance than the one of ED sample/decision statistics-based CSS scheme considered in most of the literature.

4. Design Guideline for Upgrading Sensor Networks The advantage of the proposed approach in the considered heterogeneous scenario has been further illustrated in Fig. 3(b). In this result, we plot the $P_{d}$ versus the ratio of the second generation nodes to the total number of nodes with parameters $(N=100$, $\left.N_{c}=10, S N R=-10 \mathrm{~dB}, P_{f}=0.1\right)$. Further, we consider a single antenna for the first generation sensors and multiple antennas for the second generation sensors. It can be noted from the result that as the number of the second generation nodes in the network increases, the detection performance increases and becomes more or less constant beyond a certain value of this ratio. For example, for the combination of sensors with the ME and energy decision statistics, the performance becomes constant at the ratio of 0.6. This means that $60 \%$ new nodes in the network will be sufficient to have reliable sensing performance and it's not necessary to replace all the existing nodes of the network. In other words, the network designer can choose this ratio based on the desired performance criteria and network parameters such as the number of antennas, sampling rate etc.

\section{Conclusions and Future Works}

In this paper, we have considered an interesting problem of CSS with heterogeneous nodes having different capabilities. A novel technique based on the joint PDF has been proposed in order to combine multiple decision statistics forwarded by heterogeneous cooperating nodes at the FC. The performance of the proposed technique has been compared with several existing data/decision fusion techniques. It has been noted that there exists a trade-off between the detection performance and the bandwidth overhead in the reporting channels while using various cooperative schemes and the proposed scheme provides less overhead than the overhead required by the cooperative schemes based on the sample forwarding. Further, a design guideline for the network operators has been suggested. In our future work, we target to extend this work for the CSS with more 
than two decision statistics and to explore suitable cooperative techniques for the scenarios with cooperating nodes having several heterogeneous features.

\section{Acknowledgement}

This work was supported by the National Research Fund, Luxembourg under the CORE project "SEMIGOD".

\section{References}

[1] Goldsmith, A., et al.: Breaking spectrum gridlock with cognitive radios: An information theoretic perspective. Proc. IEEE 97(5), 894 -914 (May 2009)

[2] Sharma, S.K., Chatzinotas, S., Ottersten, B.: Satellite cognitive communications: Interference modeling and techniques selection. In: 6th ASMS and 12th SPSC. pp. 111-118 (Sept 2012)

[3] Axell, E., et al.: Spectrum sensing for cognitive radio: State-of-the-art and recent advances. IEEE Signal Process. Magazine, 29(3), 101-116 (May 2012)

[4] Zeng, Y., Liang, Y.C.: Eigenvalue-based spectrum sensing algorithms for cognitive radio. IEEE Trans. Commun. 57(6), $1784-1793$ (June 2009)

[5] Chatzinotas, S., Sharma, S.K., Ottersten, B.: Asymptotic analysis of eigenvalue-based blind spectrum sensing techniques. In: IEEE ICASSP. pp. 4464-4468 (May 2013)

[6] Akyildiz, I., Lo, B., Balakrishnan, R.: Coperative spectrum sensing in cognitive radio networks: A survey. Physical Commun. 4(2011), 40-62 (2011)

[7] X. Chen, H.C., Meng, W.: Cooperative communications for cognitive radio networks-from theory to applications. IEEE Comm. Surveys Tutorials (99), 1-13 (2014)

[8] Quan, Z., et al.: Collaborative wideband sensing for cognitive radios. IEEE Signal Process. Mag. 25(6), 60-73 (Nov 2008)

[9] Teguig, D., et al.: Data fusion schemes for cooperative spectrum sensing in cognitive radio networks. In: Commun. and Info. Systems Conf., 2012 Military. pp. 1-7 (Oct 2012)

[10] Liang, Y.C., et al.: Sensing-throughput tradeoff for cognitive radio networks. IEEE Trans. Wireless Commun.,7(4), 1326-1337 (April 2008)

[11] Digham, F.F., Alouini, M.S., Simon, M.K.: On the energy detection of unknown signals over fading channels. IEEE Trans. Commun. 55(1), $21-24$ (Jan 2007)

[12] Sharma, S.K., Chatzinotas, S., Ottersten, B.: Exploiting polarization for spectrum sensing in cognitive SatComs. In: Porc. CROWNCOM. pp. 36-41 (Sept 2012)

[13] Sharma, S.K., Chatzinotas, S., Ottersten, B.: Spectrum sensing in dual polarized fading channels for cognitive satcoms. In: GLOBECOM, 2012 IEEE. pp. 3419-3424 (Dec 2012)

[14] Wang, P., et al: Multiantenna-assisted spectrum sensing for cognitive radio. IEEE Trans. Veh. Technol. 59(4), 1791-1800 (May 2010)

[15] Sharma, S.K., Chatzinotas, S., Ottersten, B.: Eigenvalue based sensing and SNR estimation for cognitive radio in presence of noise correlation. IEEE Trans. Veh. Technol. 62(8), 1-14 (Sept 2013)

[16] Sharma, S.K., Chatzinotas, S., Ottersten, B.: SNR estimation for multi-dimensional cognitive receiver under correlated channel/noise. IEEE Trans. Wireless Commun. 12(12), 6392-6405 (Dec 2013)

[17] Sharma, S.K., Chatzinotas, S., Ottersten, B.: Maximum eigenvalue detection for spectrum sensing under correlated noise. In: Proc. IEEE ICASSP. pp. 4464-4468 (May 2014)

[18] Nallagonda, S., et al.: Performance of cooperative spectrum sensing with soft data fusion schemes in fading channels. In: IEEE Annual India Conf. (INDICON), 2013 Annual IEEE. pp. 1-6 (Dec 2013)

[19] Tandra, R., Sahai, A.: SNR walls for signal detection. IEEE J. Sel. Topics Signal Porcess. 2(1), 4-17 (Feb 2008)

[20] Gatignon, H.: Statistical Analysis of Management Data, chap. 2. Springer (2010)

[21] Kay, S., Ding, Q., Emge, D.: Joint pdf construction for sensor fusion and distributed detection. In: 13th Conf. Info. Fusion (FUSION). pp. 1-6 (July 2010)

[22] Shakir, M.Z., Rao, A., Alouini, M.S.: On the decision threshold of eigenvalue ratio detector based on moments of joint and marginal distributions of extreme eigenvalues. IEEE Trans. Wireless Commun., 12(3), 974-983 (March 2013)

[23] Wickens, T.D.: Elementary Signal Detection Theory, chap. 10. Oxford University Press (2001). 